\title{
Truth-Value Semantics and Functional Extensions for Classical Logic of Partial Terms Based on Equality
}

\author{
F. Parlamento
}

\begin{abstract}
We develop a bottom-up approach to truth-value semantics for classical logic of partial terms based on equality and apply it to prove the conservativity of the addition of partial description and selection functions, independently of any strictness assumption.
\end{abstract}

0.1 Introduction We assume that the reader is familiar with the natural deduction system for classical first-order logic, conceived as the result of the direct analysis of actual mathematical reasoning, as presented by Gentzen in [3]. At the same time we ask her or him to leave aside, for a moment, the now standard classical set-theoretic formulation of the notion of logical consequence. By classical logic of partial terms based on equality we mean the standard natural deduction system, with the proviso, of a semantical nature, that not all terms are assumed to be necessarily denoting; a feature that is syntactically reflected by the restriction of the usual $\forall$-elimination and $\exists$-introduction rules, as formulated by Prawitz in [13], to variables or individual parameters only. On the other hand, that a term $t$ is denoting is expressed by the assumption $\exists x(x=t)$, for $x$ not occurring in $t$, in agreement with Quine's thesis, ${ }^{1}$ as originally proposed by Hintikka in [7] and by Leblanc and Hailperin in [11]. Truth-valued semantics has been extensively investigated by Leblanc, among others (see [8], [9], and especially [10]), who presents it as the result of a progressive simplification of the standard set-theoretic semantics, first to countable models, then to Henkin's models, and finally to no model at all. Quite the opposite, we wish to show that truth-value semantics can be approached from below, so to speak, by following the search of the simplest mathematical means by which one can establish that a proposition is not deducible from others, by the application of the given natural deduction rules, if that is indeed the case. We will explain to what extent that approach

Received April 23, 2012; accepted October 26, 2012

2010 Mathematics Subject Classification: Primary 03B20

Keywords: truth-value semantics, partial logic, equality, description and selection functions

(C) 2014 by University of Notre Dame $\quad 10.1215 / 00294527-2688078$ 
determines the usual truth tables for the propositional connectives and how it leads to truth-value semantics, when quantifiers are involved. A distinguishing feature of our treatment, with respect to Leblanc's, is that it deals with first-order languages endowed with function symbols, which, apart from its intrinsic interest, is clearly necessary if $t v$-semantics for partial logic has to be applied to show the conservativity of the addition of partial description and selection functions. As in Gentzen [4] and Prawitz [13], we refer to the articulation of a first-order language in which, beyond a countable supply of variables meant to be used for quantification, one has also an infinite supply of individual parameters meant to remain free names for generic objects of whatever (nonempty) domain one happens to be talking about. Once truthvalue semantics ( $t v$-semantics, for short) is defined, we will sketch a proof that it is indeed fully adequate, namely, that not only our motivating goal, namely, correctness, but also completeness holds. Then we establish the extension property, which will be basic for all later developments. The basic idea to deal semantically with the undefinedness of a pure term $t$ with respect to a truth-value valuation ( $t v$-valuation for short) $v$ is simply to say that $t$ is nondenoting with respect to $v$ if for all individual parameters $a, v(a=t)=\mathbf{f}$. Our main purpose is then to employ $t v$-semantics to show that the above logical framework is appropriate to deal with nonempty domains, with a language in which individual parameters stand for objects of the domain but more general terms, such as -1 or $1 /(a-a)$, when the natural or the real numbers are involved, need not denote any object whatsoever (see Feferman [2] for a more extended and very illuminating discussion). In fact, by using $t v$-semantics, we will prove the conservativity of the addition of partial selection and description functions, also when to the underlying logical framework we add the strictness axioms stating that: (1) all constants are denoting, (2) if $f t_{1} \cdots t_{n}$ is denoting, then $t_{1}, \ldots, t_{n}$ are denoting as well, and (3) for $p$ other than $=$, if $p t_{1} \cdots t_{n}$ holds, then $t_{1}, \ldots, t_{n}$ are denoting. To obtain our conservativity results, we have obviously to take into account all possible $t v$-valuations: those for which there is a nondenoting term can be disposed with by choosing one such term. For the remaining ones, to be called totally denoting $t v$-valuations, we have to enrich the language with a new constant, the undefined $\uparrow$, and show that the given valuation can be extended to the new language in a way that actually leaves $\uparrow$ undefined. To deal with the strictness axioms, we have to adopt a corresponding type of $t v$-valuation and show that the extension property applies to them as well. The conservativity of the addition of partial selection functions and partial description functions, with or without strictness axioms, then follows by a straightforward correctness/completeness argument. Finally, it is to be noted that totally denoting valuations are elementarily equivalent to classical set-theoretic structures (with total functions interpreting function symbols) and strict valuations are elementarily equivalent to set-theoretic structures with partial functions interpreting function symbols. As such, totally denoting $t v$-valuations constitute a natural intermediate step for the introduction of what has become the standard semantics for classical first-order logic, with completeness achieved as a simple corollary. Correctness, on the other hand, crucially depends on proving the substitution lemmas (which, presumably, involves the tedious details mentioned in Gumb's obituary of Leblanc [6]). ${ }^{2}$

\subsection{Pure terms and formulas}

Definition 0.1 Given a first-order language $\mathscr{L}$,

(a) a term $t$ of $\mathscr{L}$ is pure if no variable occurs in $t$; 
(b) a formula $F$ of $\mathscr{L}$ is pure if no variable occurs free in $F$.

The collection of pure terms of $\mathscr{L}$ will be denoted by PureTerm $\mathscr{L}$.

In particular, sentences are pure formulas. This terminology is inspired by Gentzen's suggestion ("rein logische Formel") in [3, p. 179] and in [5, p. 70]. ${ }^{3}$

0.3 Natural deduction systems for partial logic As for the deductive apparatus, we refer to the natural deduction system, which we denote by $N_{c}$, in which the $\forall$ elimination and $\exists$-introduction rules take the restricted form

$$
\frac{\forall x F}{F\{x / \mathbf{y}\}} \quad \frac{F\{x / \mathbf{y}\}}{\exists x F},
$$

where $\mathbf{y}$ is either a free variable or an individual parameter. A deduction is said to be pure when it involves pure formulas only, in particular in its $\forall$-elimination and $\exists$-introduction, and $\mathbf{y}$ must be a parameter. $G_{1}, \ldots, G_{n} \triangleright_{c} F$ denotes that there is a deduction in $N_{c}$ with conclusion $F$ and active assumptions included among $G_{1}, \ldots, G_{n}$.

0.4 A "bottom-up" approach to truth-value semantics At the propositional level, when required to explain why, for example, $A$ does not follow from $A \rightarrow B$ and $B$, one usually provides examples taken from ordinary or mathematical language, like letting $A$ be "the car runs out of gas" and $B$ be "the car stops," where all that is relevant is our persuasion that if $A$ is true, then $B$ is true as well, but if $B$ is true, $A$ need not necessarily be true. That naturally leads to the idea of a valuation of the propositional atoms of the propositions we are investigating into at least two values. Our goal of showing that $F$ does not follow from $G_{1}, \ldots, G_{n}$ is reached if a method of computing values for compound statements is found such that one specific value, say, $\mathbf{t}$, is preserved by deductions and a valuation $v$ of the propositional atoms in $G_{1}, \ldots, G_{n}, F$ is found, such that $G_{1}, \ldots, G_{n}$ takes the value $\mathbf{t}$ but $F$ does not.

Clearly for that to work, at least two values are needed. Classical propositional semantics makes the minimal choice of two values, say, $\mathbf{t}$ and $\mathbf{f}$. Then, as discussed, for example, by Massey in [12] and by Belnap and Massey in [1], letting $\triangleright_{\mathrm{pc}}$ be the restriction of $\triangleright_{c}$ obtained when only the application of propositional rules is allowed, the rules for $\wedge$, the introduction rules for $\vee$ and $\rightarrow$, together with the relations $A, \neg A \triangleright_{\mathrm{pc}} B$ and $A, \neg B \triangleright_{\mathrm{pc}} \neg(A \rightarrow B)$, determine the classical truth table for $\wedge$, half of the truth table for $\neg$, and three-fourths of the truth tables for $\vee$ and $\rightarrow$. On the ground of the further relations $\neg A, \neg B \triangleright_{\mathrm{pc}} \neg(A \vee B)$ and $\neg A \triangleright_{\mathrm{pc}} A \rightarrow B$, it then suffices to assume that $\neg A$ takes the value $\mathbf{t}$, whenever $A$ takes the value f, to obtain the classical truth tables. ${ }^{4}$ When it comes to quantifiers we have that $v(F\{x / a\})(F\{x / a\}$ pure $)$, for $a$ an individual parameter, has to take the value $\mathbf{t}$ whenever $v(\forall x F)$ takes the value $\mathbf{t}$, because of the $\forall$-elimination rule. Similarly $v(\exists x F)$ has to take the value $\mathbf{t}$ if for some parameter $a, v(F\{x / a\})$ takes the value $\mathbf{t}$, because of the $\exists$-introduction rule. As we will show, an appropriate solution to our problem is obtained by simply reversing the last two implications, namely, by stating that it is sufficient for $v(\forall x F)$ to take the value $\mathbf{t}$, that for every individual parameter $a$ of the language, $v(F\{x / a\})$ takes the value $\mathbf{t}$, and similarly that it is necessary for $v(\exists x F)$ to take the value $\mathbf{t}$, that for some parameter $a, v(F\{x / a\})$ takes the value $\mathbf{t}$. 


\subsection{Truth-value valuations}

Definition 0.2 Let $\mathscr{L}$ be a first-order language. A truth-value valuation ( $t v$ valuation for short) of $\mathscr{L}$ is a total function $v$ from the collection of pure atomic formulas of $\mathscr{L}$ into $\{\mathbf{t}, \mathbf{f}\}$ such that $v(\perp)=\mathbf{f}$.

A $t v$-valuation $v$ of $\mathscr{L}$ determines a unique extension $\bar{v}$ to the pure formulas of $\mathscr{L}$, according to the classical two-valued truth tables and the conditions:

- $\bar{v}(\forall x H)=\mathbf{t}$ if and only if for every parameter $a, \bar{v}(H\{x / a\})=\mathbf{t}$;

- $\bar{v}(\exists x H)=\mathbf{t}$ if and only if for some parameter $a, \bar{v}(H\{x / a\})=\mathbf{t}$.

Definition 0.3 A pure formula $F$ is $t v$-satisfied by $v$ if $\bar{v}(F)=\mathbf{t} ; F$ is $t v$-valid if every $t v$-valuation $v$ of $\mathscr{L}(F)$ satisfies $F$ and $F$ is a $t v$-semantic consequence of the pure formulas $G_{1}, \ldots, G_{n}, F$ if every $t v$-valuation $v$ of $\mathscr{L}\left(G_{1}, \ldots, G_{n}, F\right)$ which $t v$-satisfies $G_{1}, \ldots, G_{n}, t v$-satisfies $F$ as well.

0.6 Correctness and completeness for $\boldsymbol{t} \boldsymbol{v}$-semantics Correctness and completeness of the $t v$-semantics determined as above by the $t v$-valuations, for the pure system $N_{c}$, holds.

Theorem 0.1 For $G_{1}, \ldots, G_{n}, F$ pure formulas, $G_{1}, \ldots, G_{n} \triangleright_{c} F$ if and only if $F$ is a tv-semantic consequence of $G_{1}, \ldots, G_{n}$.

Proof Correctness is proved by a straightforward induction on the height of deductions in pure $N_{c}$. The only not entirely trivial case occurs when the deduction ends with a $\forall: I$ or $\exists: E$. For example in the former case, letting $\mathscr{D}$ be the immediate subderivation with conclusion $H\{x / a\}$, given any parameter $b$ of $\mathscr{L}\left(G_{1}, \ldots, G_{n}, F\right)$, if $b$ is used as proper in (some $\forall: I$ or $\exists: E$ rule applied in) $\mathscr{D}$, we first rename the occurrences of $b$ in $\mathscr{D}$ by a parameter $c$ new to $\mathscr{D}$ and then replace $a$ by $b$ throughout. The result is a deduction of $H\{x / b\}$. By the induction hypothesis, any $t v$-valuation which satisfies $G_{1}, \ldots, G_{n}$ satisfies $H\{x / b\}$ as well. But that means that it satisfies $\forall x H$, as desired. Completeness can be proved, for example, by applying the semantic tableaux method to pure formulas and considering only parameters in the $\gamma$-reductions. If $F$ is a consequence of $G_{1}, \ldots, G_{n}$, the systematic tableaux procedure, initialized with $t . G_{1}, \ldots, t . G_{n}, f . F$, returns a closed tableaux from which a deduction $\mathscr{D}$ of $F$ from $G_{1}, \ldots, G_{n}$ can be obtained. Furthermore, the variables which have bound occurrences in $\mathscr{D}$ are exactly those which occur bound in $G_{1}, \ldots, G_{n}, F$.

Note To have a correct and complete semantics for general formulas it suffices to state that $F$ is a $t v$-semantic consequence of $G_{1}, \ldots, G_{n}$ if for some substitution $\theta=\left\{x_{1} / a_{1}, \ldots, x_{n} / a_{n}\right\}$, where $x_{1}, \ldots, x_{n}$ are the variables which have free occurrences in $G_{1}, \ldots, G_{n}, F$, and $a_{1}, \ldots, a_{n}$ are distinct parameters not occurring in $G_{1}, \ldots, G_{n}, F$, we have that $F \theta$ is a $t v$-semantic consequence of $G_{1} \theta, \ldots, G_{n} \theta$. Correctness holds since from a deduction $\mathscr{D}$ of $F$ from $G_{1}, \ldots, G_{n}$, after renaming the parameters among $a_{1}, \ldots, a_{n}$, which are used as proper in $\mathscr{D}$, one obtains a deduction of $F \theta$ from $G_{1} \theta, \ldots, G_{n} \theta$, simply by replacing $x_{1}, \ldots, x_{n}$ by $a_{1}, \ldots, a_{n}$ throughout $\mathscr{D}$. As for completeness, we first note that its assumption and conclusion are invariant under renaming of bound variables. Therefore, we may assume that no variable occurs both free and bound in $G_{1}, \ldots, G_{n}, F$. Since, by assumption, $F \theta$ is a pure semantic consequence of $G_{1} \theta, \ldots, G_{n} \theta$, we may obtain a deduction 
of $F \theta$ from $G_{1} \theta, \ldots, G_{n} \theta$ in pure $N_{c}$, which is transformed into a deduction of $F$ from $G_{1}, \ldots, G_{n}$ simply by replacing $a_{1}, \ldots, a_{n}$ with $x_{1}, \ldots, x_{n}$ throughout. An immediate consequence is that the definition of $t v$-semantic consequence for general formulas does not depend on the choice of $\theta$.

0.7 Equality Following [11], as axioms for equality we take reflexivity, namely, $\forall(t=t)$, where $t$ is assumed to be parameter-free and $\forall$ denotes universal closure, and we take the axiom of substitutivity of the form

$$
\forall(r=s \rightarrow(F\{v / r\} \rightarrow F\{v / s\}))
$$

with $r, s$, and $F$ parameter-free. The two schemata of reflexivity and substitutivity will be denoted by $\mathrm{Rfl}^{=s}$ and $\mathrm{Sbst}^{=s}$. $\mathrm{Rfl}^{=s}$ and $\mathrm{Sbst}^{=s}$ are easily seen to be equivalent over $N_{c}$ to $\mathrm{Rfl}^{=s}$ and

$$
\begin{array}{ll}
\text { Symm }^{=s} & \forall(r=s \rightarrow s=r), \\
\text { Trans }^{=s} & \forall(r=s \rightarrow(s=t \rightarrow r=t)), \\
\text { Cng }_{p}^{=s} & \forall\left(r_{1}=s_{1} \wedge \cdots \wedge r_{n}=s_{n} \rightarrow\left(p\left(r_{1}, \ldots, r_{n}\right) \rightarrow p\left(s_{1}, \ldots, s_{n}\right)\right)\right), \\
\text { Cng }_{f}^{=s} & \forall\left(r_{1}=s_{1} \wedge \cdots \wedge r_{n}=s_{n} \rightarrow f\left(r_{1}, \ldots, r_{n}\right)=f\left(s_{1}, \ldots, s_{n}\right)\right),
\end{array}
$$

for any $n$-ary relation and function symbols $p$ and $f$, where all the terms shown are parameter-free. $N_{c}^{=}$results from $N_{c}$ by allowing any formula in $\mathrm{Rfl}^{s}$ and $\mathrm{Sbst}^{s}$ to be considered as a discharged assumption.

Note The fact that the equality axioms, formulated for variables only, namely, $\forall x(x=x)$ and $\forall x \forall y(x=y \rightarrow(F\{v / x\} \rightarrow F\{v / y\}))$, are not sufficient for a satisfactory development of the logic of partial terms was first noticed in [11].

\section{$0.8 t v$-semantics for $N_{c}^{=}$}

Definition 0.4 A $t v$-valuation with equality of $\mathscr{L}$ is a $t v$-valuation of $\mathscr{L}$, which satisfies the axioms in $\mathrm{Rfl}^{=}, \mathrm{Symm}^{=s}$, Trans $=s$, and $\mathrm{Cng}^{=s}$.

In other words, $v$ is a $t v$-valuation with equality if the binary relation $\{(r, s)$ : $v(r=s)=\mathbf{t}\}$, to be denoted by $=^{v}$, is a congruence relation with respect to the canonical interpretation of the function symbols $\left\{\left(\left(t_{1}, \ldots, t_{n}\right), f\left(t_{1}, \ldots, t_{n}\right)\right)\right\}$ and the relations $p^{v}=\left\{\left(t_{1}, \ldots, t_{n}\right): v\left(p\left(t_{1}, \ldots, t_{n}\right)\right)=\mathbf{t}\right\}$, for $p$ a relation symbol in $\mathscr{L}$, where $t_{1}, \ldots, t_{n}$ range over PureTerm $\mathscr{L}$.

Correctness and completeness for $N_{c}^{=}$holds with respect to the notion of $t v$ semantic consequence based on $t v$-valuations with equality.

Theorem 0.2 For $G_{1}, \ldots, G_{n}, F$ pure formulas, $G_{1}, \ldots, G_{n} \triangleright_{c}^{=} F$ if and only if every $t v$-valuation with equality of $\mathscr{L}\left(G_{1}, \ldots, G_{n}, F\right)$ which $t v$-satisfies $G_{1}, \ldots, G_{n}$, tv-satisfies $F$ as well.

Proof Correctness is an immediate consequence of the correctness of $N_{c}$. Completeness can be achieved through the tableaux method by interleaving the logical reduction steps with steps in which one appends, one after the other, the countably many judgments of the form $t . E$, where $E$ belongs to $\mathrm{Rfl}^{=s}$, Symm ${ }^{=s}$, Trans ${ }^{=s}$, or $\mathrm{Cng}^{=s}$.

Extension to general formulas can be obtained as for $N_{c}$. 
0.9 The extension property The following property will be our basic tool for dealing with $t v$-semantics for $N_{c}$ and $N_{c}=$.

Proposition 0.1 (Extension property) If $v$ is a $t v$-valuation of $\mathscr{L}$ (with equality) and $\mathscr{L} \subset \mathscr{L}^{\prime}$, then there is a map $\Phi$ from PureTerm $\mathscr{L}^{\prime}$ onto PureTerm $\mathscr{L}$ and a valuation (with equality) $v^{\prime}$ of $\mathscr{L}^{\prime}$ such that

(1) for a term $t$ of $\mathscr{L}$ with variables among $x_{1}, \ldots, x_{k}$ and pure terms $r_{1}^{\prime}, \ldots, r_{k}^{\prime}$ of $\mathscr{L}^{\prime}$,

$$
\Phi\left(t\left\{x_{1} / r_{1}^{\prime}, \ldots, x_{k} / r_{k}^{\prime}\right\}\right)=t\left\{x_{1} / \Phi\left(r_{1}^{\prime}\right), \ldots, x_{k} / \Phi\left(r_{k}^{\prime}\right)\right\},
$$

in particular if $t$ is a pure term of $\mathscr{L}, \Phi(t)=t$;

(2) for a formula $F$ of $\mathscr{L}$ with free variables among $x_{1}, \ldots, x_{k}$ and pure terms $r_{1}^{\prime}, \ldots, r_{k}^{\prime}$ of $\mathscr{L}^{\prime}$

$$
\bar{v}^{\prime}\left(F\left\{x_{1} / r_{1}^{\prime}, \ldots, x_{k} / r_{k}^{\prime}\right\}\right)=\bar{v}\left(F\left\{x_{1} / \Phi\left(r_{1}^{\prime}\right), \ldots, x_{k} / \Phi\left(r_{k}^{\prime}\right)\right\}\right) ;
$$

in particular, if $F$ is a pure formula of $\mathscr{L}$, then $\bar{v}^{\prime}(F)=\bar{v}(F)$.

Proof For every $n$-ary function symbol $f \in \mathscr{L}^{\prime} \backslash \mathscr{L}$, fix a total function f : PureTerm $_{\mathscr{L}}^{n} \rightarrow$ PureTerm $_{\mathscr{L}}$ (for $n=0, f$ is either a constant or a parameter and f is a pure term, say, $f_{0}$, of $\mathscr{L}$ ), which, in case $v$ is a $t v$-valuation with equality, is congruent with respect to $={ }^{v}$ (e.g., $\mathbf{f}$ can be any constant function). If $t$ is a parameter or a constant of $\mathscr{L}$, let $\Phi(t)=t$. If $t^{\prime}$ is a parameter or a constant in $\mathscr{L}^{\prime} \backslash \mathscr{L}$, let $\Phi\left(t^{\prime}\right)=t_{0}^{\prime}$. If $t^{\prime}$ is $g\left(t_{1}^{\prime}, \ldots, t_{n}^{\prime}\right)$ with $g$ in $\mathscr{L}$, let $\Phi\left(t^{\prime}\right)=g\left(\Phi\left(t_{1}^{\prime}\right), \ldots, \Phi\left(t_{n}^{\prime}\right)\right)$. Finally, if $t^{\prime}$ is $f\left(t_{1}^{\prime}, \ldots, t_{n}^{\prime}\right)$, let $\Phi\left(t^{\prime}\right)=\mathbf{f}\left(\Phi\left(t_{1}^{\prime}\right), \ldots, \Phi\left(t_{n}^{\prime}\right)\right)$. Furthermore for $p$, an $n$-ary relation symbol of $\mathscr{L}$, let

$$
v^{\prime}\left(p\left(t_{1}^{\prime}, \ldots, t_{n}^{\prime}\right)\right)=v\left(p\left(\Phi\left(t_{1}^{\prime}\right), \ldots, \Phi\left(t_{n}^{\prime}\right)\right)\right)
$$

and, for $q$, an $n$-ary relation symbol in $\mathscr{L}^{\prime} \backslash \mathscr{L}$, let $v^{\prime}\left(q\left(t_{1}^{\prime}, \ldots, t_{n}^{\prime}\right)\right)$ be defined arbitrarily provided that $v^{\prime}\left(q\left(s_{1}^{\prime}, \ldots, s_{n}^{\prime}\right)\right)=\mathbf{t}$, whenever $v^{\prime}\left(q\left(t_{1}^{\prime}, \ldots, t_{n}^{\prime}\right)\right)=\mathbf{t}$ and $v^{\prime}\left(t_{1}^{\prime}=s_{1}^{\prime}\right)=\mathbf{t}, \ldots, v^{\prime}\left(t_{n}^{\prime}=s_{n}^{\prime}\right)=\mathbf{t}$.

Both (1) and (2) are easily proved by induction on the height of $t$ and $F$, respectively.

Since, in the previous proof, it is the choice of $\mathbf{f}$ which determines $v^{\prime}$, we will say that $v^{\prime}$ is the extension of $v$ based on $\mathbf{f}$.

Remark The notion of $t v$-valuation can be relativized to any fixed subset $\mathcal{P}_{0}$ of the set of parameters of $\mathscr{L}$, assumed to be nonempty, in the case when $\mathscr{L}$ has no constant, by taking into account only the formulas whose parameters belong to $\mathcal{P}_{0}$ and considering only parameters in $\mathcal{P}_{0}$ in defining the meaning of the quantifiers. If $\mathcal{P}_{0}$ is infinite, the proof of correctness remains unchanged. If $\mathcal{P}_{0}$ is finite, correctness can be established along the lines of the previous proof. In fact, if $v_{0}$ is a valuation restricted to any set of parameters $\mathcal{P}_{0}$ which satisfies $F$, then it suffices to note that $v_{0}$ can be extended to a valuation $v_{0}^{\prime}$ of $\mathscr{L}(F)$, which still satisfies $F$, by mapping all the parameters which do not belong to $\mathcal{P}_{0}$ into any one of the parameters in $\mathcal{P}_{0}$.

Thus, for example, the $t v$-valuation restricted to $\{a, b\}$,

$$
\begin{aligned}
& \{(p(a, a), \mathbf{t}),(p(b, b), \mathbf{t}),(p(a, b), \mathbf{f}),(p(b, a), \mathbf{f}),((a=a), \mathbf{t}), \\
& \quad((b=b), \mathbf{t}),((a=b), \mathbf{f}),((b=a), \mathbf{f})\}
\end{aligned}
$$


which satisfies $\forall x \exists y p(x, y)$, but does not satisfy $\exists x \forall y p(x, y)$, suffices to show that in $N_{c}^{=}$one cannot deduce the latter sentence from the former. Similarly the $t v$-valuation restricted to $\{a\}$,

$$
\{(p(c), \mathbf{t}),(p(a), \mathbf{f}),(a=a, \mathbf{t}),(c=c, \mathbf{t}),(a=c, \mathbf{f}),(c=a, \mathbf{f})\},
$$

for $c$ a constant, suffices to show that in $N_{c}^{=}, \exists x p(x)$ cannot be deduced from $p(c)$, and the $t v$-valuation restricted to $\{a\}$,

$$
\begin{aligned}
\left\{\left(p\left(f^{n}(a), f^{n+1}(a)\right), \mathbf{t}\right): n \in N\right\} & \cup\left\{\left(p\left(f^{n}(a), f^{m}(a)\right), \mathbf{f}\right): m \neq n+1\right\} \\
\cup\left\{\left(f^{n}(a)=f^{n}(a), \mathbf{t}\right): n \in N\right\} & \cup\left\{\left(f^{n}(a)=f^{m}(a), \mathbf{f}\right): n \neq m\right\},
\end{aligned}
$$

where $f^{0}(a)$ denotes $a$ itself, suffices to show that $\forall x \exists y p(x, y)$ is not deducible from $\forall x p(x, f(x))$. On the other hand, completeness for $t v$-valuations restricted to finite sets of parameters clearly fails. For example, $\exists x p(x, x)$ is not derivable in $N_{c}^{=}$from $\forall x \exists y p(x, y)$ and $\forall x \forall y \forall z(p(x, y) \wedge p(y, z) \rightarrow p(x, z))$, although it is satisfied by any $t v$-valuation restricted to a finite set of parameters, which satisfies the latter two sentences.

\subsection{Totally denoting valuations}

Notation $\quad t \downarrow$ denotes the formula $\exists y y=t$, for $y$ any variable not occurring in $t$.

The usual natural deduction system with equality, in which $\forall$-elimination and $\exists$-introduction can be applied to any substitutable term, is easily seen to be equivalent to $N_{c}^{=}$, provided that $\forall(t \downarrow)$ is allowed as a discharged assumption, for any term $t$. We denote with $N_{c}^{\downarrow=}$ the resulting deduction system. $N_{c}^{\downarrow}=$ is clearly equivalent to $N_{c}^{=}$, provided that formulas of the form $c \downarrow$ and $\forall x_{1}, \ldots, x_{n} f\left(x_{1}, \ldots, x_{n}\right) \downarrow$, for all the constant $c$ and function symbol $f$ of the language, are allowed as discharged assumptions.

Definition 0.5 A $t v$-valuation with equality $v$ for $\mathscr{L}$ is said to be totally denoting if for every pure term $t$ of $\mathscr{L}, v t v$-satisfies $t \downarrow$; namely, there is a parameter $a$ such that $v(a=t)=\mathbf{t}$.

Proposition 0.2 A tv-valuation $v$ for $\mathscr{L}$ with equality is totally denoting if and only if every constant of $\mathscr{L}$ is denoting, and for every $n$-ary function symbol $f$ and $n$-tuple of parameters $a_{1}, \ldots, a_{n}, f\left(a_{1}, \ldots, a_{n}\right)$ is denoting.

Proof The proposition is proved by a straightforward induction on the height of terms.

Theorem 0.3 Correctness and completeness for $N_{c}^{\downarrow=}$ holds with respect to the notion of t $v$-semantic consequence based on totally denoting t $v$-valuations.

Proof The proof is immediate from the above propositions.

Note To every totally denoting $t v$-valuation $v$ for $\mathscr{L}$ there corresponds an elementarily equivalent set-theoretic interpretation $I_{v}$. The domain $D^{I_{v}}$ of $I_{v}$ is the set of parameters of $\mathscr{L}$. The interpretation of a constant symbol in $I_{v}$ is a parameter $a$, 
such that $v(a=c)=\mathbf{t}$. Similarly the interpretation of an $n$-ary function symbol $f$ is the total function:

$$
f^{I_{v}}=\left\{\left(\left(a_{1}, \ldots, a_{n}\right), b\right): v\left(b=f\left(a_{1}, \ldots, a_{n}\right)\right)=\mathbf{t}\right\} .
$$

Finally, for any relation symbol $p$ of $\mathscr{L}$,

$$
p^{I_{v}}=\left\{\left(a_{1}, \ldots, a_{n}\right): v\left(p\left(a_{1}, \ldots, a_{n}\right)\right)=\mathbf{t}\right\} .
$$

Let $\tau$ be any assignment of elements of $D^{I_{v}}$ to variables and parameters which leaves all the parameters fixed, so that, under $\tau$, the value of any pure term $t$ is $t$ itself. A straightforward induction shows that if $F$ is a pure formula of $\mathscr{L}$, then $\bar{v}(F)=\mathbf{t}$ if and only if $I_{v}, \tau \models F$. As a consequence, for every sentence $F$ of $\mathscr{L}, \bar{v}(F)=\mathbf{t}$ if and only if $I_{v} \models F$, which is what we mean by saying that $v$ and $I_{v}$ are elementarily equivalent. The quotient of $I_{v}$ with respect to $={ }^{v}$ is a normal structure elementarily equivalent to $I_{v}$ and therefore to $v$. The completeness theorem for (the ordinary settheoretic semantics of) $N_{c}^{\downarrow=}$ is thus an immediate consequence of the completeness of $t v$-semantics with equality for $N_{c}^{\downarrow}$.

Proposition 0.3 The extension property holds also for the totally denoting valuations.

Proof If $v$ is totally denoting and $v^{\prime}$ is an extension of $v$ to $\mathscr{L}^{\prime}$, then $v^{\prime}$ is also totally denoting since $\bar{v}^{\prime}\left(\exists x\left(x=t^{\prime}\right)\right)=\bar{v}\left(\exists x\left(x=\Phi\left(t^{\prime}\right)\right)\right.$ and $\bar{v}\left(\exists x\left(x=\Phi\left(t^{\prime}\right)\right)=\mathbf{t}\right.$, because $\Phi\left(t^{\prime}\right)$ is a pure term of $\mathscr{L}$ and $v$ is totally denoting.

\subsection{Introducing the undefined $\uparrow$}

Proposition 0.4 A totally denoting $t v$-valuation $v$ of $\mathscr{L}$ can be extended to a $t v$ valuation $v^{\uparrow}$ with equality of the language $\mathscr{L}+\uparrow$, where $\uparrow$ is a constant not belonging to $\mathscr{L}$, such that for every pure formula $F$ of $\mathscr{L}, \bar{v}(F)=\bar{v}^{\uparrow}(F)$ and $\uparrow$ is nondenoting with respect to $v^{\uparrow}$.

Proof We set $v^{\uparrow}(r=s)=\mathbf{t}$ if and only if $r=s$ belongs to the smallest set of equalities between pure terms of $\mathscr{L}+\uparrow$, which contains all the equalities $t^{\prime}=t^{\prime}$ and $r=s$ such that $v(r=s)=\mathbf{t}$ and furthermore contains $f\left(r_{1}, \ldots, r_{n}\right)=f\left(s_{1}, \ldots, s_{n}\right)$ whenever for all $1 \leq i \leq n$ it already contains $r_{i}=s_{i}$. On all the remaining pure atomic formulas which contain $\uparrow, v^{\uparrow}$ takes the value $\mathbf{f}$ and $v^{\uparrow}(A)=v(A)$ for every pure atomic formula of $\mathscr{L}$. The claim follows by a straightforward induction on the height of $F$. To prove that $v^{\uparrow}$ is a valuation with equality it suffices to show that $v^{\uparrow}\left(r_{1}=s_{1} \wedge \cdots \wedge r_{n}=s_{n} \rightarrow\left(p\left(r_{1}, \ldots, r_{n}\right) \rightarrow\right.\right.$ $\left.\left.p\left(s_{1}, \ldots, s_{n}\right)\right)\right)=\mathbf{t}$. If all of $r_{1}, \ldots, s_{n}$ belong to $\mathscr{L}$, that holds since $v^{\uparrow}$ agrees with $v$, which is a $t v$-valuation with equality. Thus let us assume that, for example, $\uparrow$ occurs in $s_{i}$. Then, by definition, $v^{\uparrow}\left(p\left(s_{1}, \ldots, s_{n}\right)\right)=\mathbf{f}$, and we have to show that also $v^{\uparrow}\left(p\left(r_{1}, \ldots, r_{n}\right)\right)=\mathbf{f}$. That follows from the fact that if $\uparrow$ occurs in $s_{i}$ and $v^{\uparrow}\left(r_{i}=s_{i}\right)=\mathbf{t}$, then $\uparrow$ occurs also in $r_{i}$. As a matter of fact we have that if $v^{\uparrow}(r=s)=\mathbf{t}$ and $\uparrow$ occurs in $s$, then $\uparrow$ occurs also in $r$ and conversely, as it follows immediately from the definition of $v^{\uparrow}$ on equalities. Obviously, that also guarantees that $\uparrow$ cannot be denoting. 


\subsection{Strictness}

Definition 0.6 Let $N_{c}^{=s}$ be the result of adding to $N_{c}^{=}$the following strictness axioms:

(1) $c \downarrow$

(2) $\forall\left(f\left(t_{1}, \ldots, t_{n}\right) \downarrow \rightarrow t_{1} \downarrow \wedge \cdots \wedge t_{n} \downarrow\right)$,

(3) $\forall\left(p\left(t_{1}, \ldots, t_{n}\right) \rightarrow t_{1} \downarrow \wedge \cdots \wedge t_{n} \downarrow\right)$ for every relation symbol $p$ other than $=$, and $t_{1}, \ldots, t_{n}$ parameter-free.

A strict $t v$-valuation of $\mathscr{L}$ is a $t v$-valuation of $\mathscr{L}$ with equality which satisfies the strictness axioms.

In (3) we have to leave aside $=$, since otherwise, from the adoption of $t=t$ as an axiom, it would follow that every $t$ is defined. Thus our notion of strictness is more relaxed than the one usually adopted when the existence predicate is taken as primitive (see, e.g., [2]).

The proof of correctness and completeness of the semantics based on totally denoting $t v$-valuations for $N_{c}^{=}$can be easily adapted to establish the following.

Theorem 0.4 Correctness and completeness for $N_{c}^{=s}$ holds with respect to the notion of t $v$-semantic consequence based on strict t $v$-valuations.

Proposition $0.5 \quad$ The extension property holds also for strict tv-valuations, provided that the extension is based on functions $\mathbf{f}$ which are strict, namely, satisfy the following condition:

(a) if $v\left(\mathbf{f}\left(r_{1}, \ldots, r_{n}\right) \downarrow\right)=\mathbf{t}$, then $v\left(r_{1} \downarrow\right)=\mathbf{t}, \ldots, v\left(r_{n} \downarrow\right)=\mathbf{t}$.

Proof If $v$ is strict and $v^{\prime}$ is an extension of $v$ to $\mathscr{L}^{\prime}$ based on a function $\mathbf{f}$ satisfying condition $(a)$, then $v^{\prime}$ is also strict. For, assume $\bar{v}^{\prime}\left(f\left(t_{1}^{\prime}, \ldots, t_{n}^{\prime}\right) \downarrow\right)=\mathbf{t}$, namely, $\bar{v}^{\prime}\left(\exists x\left(x=f\left(t_{1}^{\prime}, \ldots, t_{n}^{\prime}\right)\right)\right)=\mathbf{t}$. If $f \in \mathscr{L}^{\prime} \backslash \mathscr{L}$ by the extension property it follows that $\bar{v}\left(\exists x\left(x=\mathbf{f}\left(\Phi\left(t_{1}^{\prime}\right), \ldots, \Phi\left(t_{n}^{\prime}\right)\right)\right)\right)=\mathbf{t}$. By the strictness of $\mathbf{f}$, it follows that $\Phi\left(t_{1}^{\prime}\right) \downarrow, \ldots, \Phi\left(t_{n}^{\prime}\right) \downarrow$, namely, $\bar{v}\left(\exists x_{1}\left(x_{1}=\Phi\left(t_{1}^{\prime}\right)\right)\right)=\mathbf{t}, \ldots$, $\bar{v}\left(\exists x_{n}\left(x_{1}=\Phi\left(t_{n}^{\prime}\right)\right)\right)=\mathbf{t}$, from which, by the extension property again, we may conclude that $\bar{v}^{\prime}\left(\exists x_{1}\left(x_{1}=t_{1}^{\prime}\right)\right)=\mathbf{t}, \ldots, \bar{v}^{\prime}\left(\exists x_{n}\left(x_{n}=t_{n}^{\prime}\right)\right)=\mathbf{t}$, namely, $\bar{v}^{\prime}\left(t_{1}^{\prime} \downarrow\right)=\mathbf{t}, \ldots, \bar{v}^{\prime}\left(t_{n}^{\prime} \downarrow\right)=\mathbf{t}$, as required for $v^{\prime}$ to be strict. The case in which $f \in \mathscr{L}$ or $\bar{v}^{\prime}\left(p\left(t_{1}^{\prime}, \ldots, t_{n}^{\prime}\right)\right)=\mathbf{t}$, for $p$ other than $=$, is entirely similar.

Note As for totally denoting $t v$-valuations, to every strict valuation $v$ of $\mathscr{L}$ there corresponds an elementarily equivalent (partial) set-theoretic interpretation $I_{v}$ of $\mathscr{L}$. $D^{I_{v}}$ is still the set of parameters of $\mathscr{L}$, but $f^{I_{v}}$ is, in general, a partial function. For a given assignment $\sigma$ of elements of $D^{I_{v}}$ to variables and parameters, the value $\sigma(t)$ which $t$ takes under $\sigma$ is an element of $D^{I_{v}}$ if and only if $t \sigma$ is a denoting term, namely, $v(t \sigma \downarrow)=\mathbf{t}$. $I, \sigma \models F$ is defined by letting $I, \sigma \models r=s$ if and only if $v(\sigma(r), \sigma(s))=\mathbf{t}$ (even if $\sigma(r)$ or $\sigma(s)$ does not belong to $D^{I_{v}}$ ); for $p$ other than $=, I_{v}, \sigma \models p\left(t_{1}, \ldots, t_{n}\right)$ if and only if $\sigma\left(t_{1}\right), \ldots, \sigma\left(t_{n}\right)$ belong to $D^{I_{v}}$ and $\left(\sigma\left(t_{1}\right), \ldots, \sigma\left(t_{n}\right)\right) \in p^{I_{v}}$ (namely, $v\left(p\left(\sigma\left(t_{1}\right), \ldots, \sigma\left(t_{n}\right)\right)=\mathbf{t}\right)$ ). For compound formulas $I_{v}, \sigma \models F$ is defined as usual. For every pure formula $F$ of $\mathscr{L}$ and assignment $\tau$, which leaves the parameters fixed, $\bar{v}(F)=\mathbf{t}$ if and only if $I_{v}, \tau \models F$, so that for a sentence $F, \bar{v}(F)=\mathbf{t}$ if and only if $I_{v} \models F$. For, if $F$ is of the form $p\left(t_{1}, \ldots, t_{n}\right)$, from $\bar{v}(F)=\mathbf{t}$, by the strictness of $v$, it follows that $t_{1}, \ldots, t_{n}$ are all denoting terms, so that $\tau\left(t_{1}\right), \ldots, \tau\left(t_{n}\right)$ belong to $D^{I_{v}}$, and $\left(\tau\left(t_{1}\right), \ldots, \tau\left(t_{n}\right)\right) \in p^{I_{v}}$ 
so that $I_{v}, \tau \models F$. As a consequence, we have the completeness of $N_{c}^{=s}$ with respect to partial set-theoretic interpretations.

Note If a $t v$-valuation $v$ is extended into $v^{\downarrow}$, rather than into $\bar{v}$, by using the clauses

(a) $v^{\downarrow}(\forall x H)=\mathbf{t}$ if and only if for every pure term $t, v^{\downarrow}(H\{x / t\})=\mathbf{t}$,

(b) $v^{\downarrow}(\exists x H)=\mathbf{t}$ if and only if for some pure term $t, v^{\downarrow}(H\{x / t\})=\mathbf{t}$,

then a straightforward modification of the previous arguments shows that the resulting semantics is correct and complete with respect to the usual natural deduction system, without equality, in which $\forall$-elimination and $\exists$-introduction can be applied to any substitutable term, to be denoted by $N_{c}^{\downarrow}$, and that the extension property still holds. Furthermore, $v^{\downarrow}$ is elementarily equivalent to a (total) set-theoretic structure $I_{v \downarrow}$, whose domain is the set $D^{I_{v}}$ of the pure terms of the language, so that the usual completeness theorem for $N_{c}^{\downarrow}$ immediately follows. The same applies if $v$ is a valuation with equality, thus obtaining a correct and complete semantics for $N_{c}^{\downarrow=}$. Since if $v$ is a totally denoting valuation, then obviously $\bar{v}=v^{\downarrow}$, the $t v$-semantics for $N_{c}^{\downarrow}$ based on $v^{\downarrow}$ subsumes the one based on totally denoting $t v$-valuations, so that its completeness can also be inferred from the completeness of the latter. As in the previous case, one can also immediately infer the usual completeness theorem for $N_{c}^{\downarrow}=$. That shows the interest of $t v$-semantics even if one is concerned only with total classical logic with or without equality. In particular, the standard classical set-theoretic semantics can be rather effectively introduced as a very natural generalization of $t v$-totally denoting semantics, by replacing the fixed domain of the pure terms of the language by an arbitrary nonempty set and the total canonical interpretation of the function symbols by their interpretation with arbitrary total functions on such a set. Concerning the last point, we wish to note the difficulty one faces in motivating the choice of totality if the classical set-theoretic structures are to be presented as a model of ordinary mathematical structures, which may carry partial, rather than only total, operations, like the reals with the $x^{-1}$ or $\log x$ function, for example.

\section{Conservativeness of Partial Selection Functions}

Theorem 1.1 If $D$ is a formula of $\mathscr{L}$ with distinct free variables $x_{1}, \ldots, x_{n}, y$, and $f$ is an $n$-ary function symbol not in $\mathscr{L}$, then the conjunction of the following two formulas is conservative over $\mathscr{L}$ with respect to $N_{c}^{=}$:

$$
\begin{aligned}
& \epsilon_{y}^{1}(f ; D) \forall\left(f\left(x_{1}, \ldots, x_{n}\right) \downarrow \rightarrow \exists y D\right), \\
& \epsilon_{y}^{2}(f ; D) \forall\left(\exists y D \rightarrow \exists y\left(y=f\left(x_{1}, \ldots, x_{n}\right) \wedge D\right)\right) ;
\end{aligned}
$$

namely, if $G_{1}, \ldots, G_{n}, F$ are formulas of $\mathscr{L}$ and $f$ does not occur in $G_{1}, \ldots, G_{n}, F$, and $G_{1}, \ldots, G_{n}, \epsilon_{y}^{1}(f ; D), \epsilon_{y}^{2}(f ; D) \triangleright_{c}^{=} F$, then $G_{1}, \ldots, G_{n} \triangleright_{c}^{=} F$. The same holds for $N_{c}^{=s}$.

Proof We deal first with the case in which $G_{1}, \ldots, G_{n}, F$ are pure. By the correctness and completeness of the $t v$-semantics with equality for $N_{c}^{=}$, it suffices to show that the extension property can be applied to any $t v$-valuation with equality $v$ of $\mathscr{L}$ so as to obtain a valuation $v^{\prime}$ of $\mathscr{L}+f$ which satisfies $\epsilon_{y}^{1}(f ; D)$ and 
$\epsilon_{y}^{2}(f ; D)$. If $v$ is not totally denoting, fix a nondenoting term $t_{0}$ of $\mathscr{L}$ and an enumeration of all the parameters of $\mathscr{L}$. If $t_{1}, \ldots, t_{n}$ are all denoting terms of $\mathscr{L}, a_{i}$ is the first parameter in the fixed enumeration such that $v\left(a_{i}=t_{i}\right)=\mathbf{t}$ and $b$ is the first one such that $v\left(D\left\{x_{1} / a_{1}, \ldots, x_{n} / a_{n}, y / b\right\}\right)=\mathbf{t}$; provided that there is such a $b$, we let $\mathbf{f}\left(t_{1}, \ldots, t_{n}\right)=b$; if on the contrary there is no $b$ such that $v\left(D\left\{x_{1} / a_{1}, \ldots, x_{n} / a_{n}, y / b\right\}\right)=\mathbf{t}$ or for some $1 \leq i \leq n, t_{i}$ is nondenoting, then we let $\mathbf{f}\left(t_{1}, \ldots, t_{n}\right)=t_{0}$. As it is easy to check, $\mathbf{f}$ is congruent with respect to $={ }^{v}$, so that the extension $v^{\prime}$ of $v$ to $\mathscr{L}+f$, based on $\mathbf{f}$, is a $t v$-valuation with equality, and it is also strict. Furthermore, $\bar{v}^{\prime}$ satisfies $\epsilon_{y}^{1}(f ; D)$ and $\epsilon_{y}^{2}(f ; D)$. Since $\epsilon_{y}^{1}(f ; D)$ follows in $N_{c}^{=}$from $\forall x_{1} \ldots \forall x_{n} \forall y\left(f\left(x_{1}, \ldots, x_{n}\right)=y \rightarrow D\right)$, it suffices to verify that $\bar{v}^{\prime}$ satisfies the last formula, namely, that for every $(n+1)$-tuple of parameters $a_{1}, \ldots, a_{n}, b$, if $\bar{v}^{\prime}\left(f\left(a_{1}, \ldots, a_{n}\right)\right)=b$, then $\bar{v}^{\prime}\left(D\left\{x_{1} / a_{1}, \ldots, x_{n} / a_{n}, y / b\right\}\right)=\mathbf{t}$. By the extension property, $\bar{v}^{\prime}\left(f\left(a_{1}, \ldots, a_{n}\right)=b\right)=\bar{v}\left(\mathbf{f}\left(a_{1}, \ldots, a_{n}\right)=b\right)$. Thus from $\bar{v}^{\prime}\left(f\left(a_{1}, \ldots, a_{n}\right)=b\right)=\mathbf{t}$ it follows that $\bar{v}\left(\mathbf{f}\left(a_{1}, \ldots, a_{n}\right)=b\right)=\mathbf{t}$, which, by the definition of $\mathbf{f}$, can only happen if $\bar{v}\left(D\left\{x_{1} / a_{1}, \ldots, x_{n} / a_{n}, y / b\right\}\right)=\mathbf{t}$.

As for $\epsilon_{y}^{2}(f ; D)$, we have to verify that for every $n$-tuple of parameters $a_{1}, \ldots, a_{n}$, if $\bar{v}^{\prime}\left(\exists y D\left\{x_{1} / a_{1}, \ldots, x_{n} / a_{n}\right\}\right)=\mathbf{t}$, then $\bar{v}^{\prime}\left(\exists y\left(y=f\left(a_{1}, \ldots, a_{n}\right) \wedge D\left\{x_{1} / a_{1}, \ldots\right.\right.\right.$, $\left.\left.\left.x_{n} / a_{n}\right\}\right)\right)=\mathbf{t}$. From the assumption, by the extension property it follows that $\bar{v}\left(\exists y D\left\{x_{1} / a_{1}, \ldots, x_{n} / a_{n}\right\}\right)=\mathbf{t}$. Thus there is a parameter $b$, which we may assume is the first in the given enumeration, such that $\bar{v}\left(D\left\{x_{1} / a_{1}, \ldots, x_{n} / a_{n}, y / b\right\}\right)=\mathbf{t}$. Therefore $\mathbf{f}\left(a_{1}, \ldots, a_{n}\right)=b$. On the other hand, $\bar{v}^{\prime}\left(\exists y\left(y=f\left(a_{1}, \ldots, a_{n}\right) \wedge\right.\right.$ $\left.\left.D\left\{x_{1} / a_{1}, \ldots, x_{n} / a_{n}\right\}\right)\right)=\mathbf{t}$ if and only if there is a parameter $c$ such that $\bar{v}^{\prime}\left(c=f\left(a_{1}, \ldots, a_{n}\right) \wedge D\left\{x_{1} / a_{1}, \ldots, x_{n} / a_{n}, y / c\right\}\right)=\mathbf{t}$. By the extension property that holds if and only if there is a parameter $c$ such that $\bar{v}\left(c=\mathbf{f}\left(a_{1}, \ldots, a_{n}\right) \wedge\right.$ $\left.D\left\{x_{1} / a_{1}, \ldots, x_{n} / a_{n}, y / c\right\}\right)=\mathbf{t}$. Therefore it suffices to take $b$ for $c$ to conclude that our claim holds. If $v$ is totally denoting, it suffices to consider its extension with the "undefinite" $v^{\uparrow}$ and replace $t_{0}$ by $\uparrow$ in the previous argument, to obtain the desired extension of $v$. By the extension property for strict valuation the result applies to $N_{c}^{=s}$ as well. To extend the result to general formulas it suffices to repeat the argument given for the extension of the completeness theorem.

\subsection{Conservativity of partial description functions}

Theorem 1.2 If $D$ is a formula of $\mathscr{L}$ with distinct free variables $x_{1}, \ldots, x_{n}, y$, and $f$ is an $n$-ary function symbol not in $\mathscr{L}$, then the following formula is conservative over $\mathscr{L}$ with respect to $N_{c}^{=}$:

$$
\iota_{y}(f ; D) \forall\left(f\left(x_{1}, \ldots, x_{n}\right)=y \equiv D \wedge \forall y^{\prime}\left(D\left\{y / y^{\prime}\right\} \rightarrow y^{\prime}=y\right)\right) .
$$

The same holds for $N_{c}^{=s}$.

Proof Given $D$, let $D^{!}$be $D \wedge \forall y^{\prime}\left(D\left\{y / y^{\prime}\right\} \rightarrow y^{\prime}=y\right)$. By the proof of the first part of Theorem 1.1 applied to $D^{!}$, we can conservatively add the formula $(a) \forall\left(f\left(x_{1}, \ldots, x_{n}\right)=y \rightarrow D^{!}\right)$. Furthermore, we can conservatively add $\epsilon_{y}^{2}\left(f ; D^{!}\right)$. From $D^{!}$it logically follows that $\exists y\left(D \wedge \forall y^{\prime}\left(D\left\{y / y^{\prime}\right\} \rightarrow y^{\prime}=y\right)\right)$, from which by $\epsilon_{y}^{2}\left(f ; D^{!}\right)$it follows that $\exists y\left(y=f\left(x_{1}, \ldots, x_{n}\right) \wedge D \wedge \forall y^{\prime}(D\{y /\right.$ $\left.\left.\left.y^{\prime}\right\} \rightarrow y^{\prime}=y\right)\right)$. Let then $z$ be such that $z=f\left(x_{1}, \ldots, x_{n}\right) \wedge D\{y / z\} \wedge \forall y^{\prime}(D\{y /$ $\left.\left.y^{\prime}\right\} \rightarrow y^{\prime}=z\right)$. From $z=f\left(x_{1}, \ldots, x_{n}\right) \wedge D\{y / z\}$ by $D^{!}$it follows that $z=y$; hence $f\left(x_{1}, \ldots, x_{n}\right)=y$. Thus also the reverse implication in $(a)$, and therefore 
$\iota_{y}(f ; D)$, is deducible in the conservative extension provided by Theorem 1.1 with respect to $D^{!}$. Hence $\iota_{y}(f ; D)$ is conservative over $\mathscr{L}$ with respect to $N_{c}^{=}$.

Corollary 1.1 Under the assumption of Theorem 1.2,

$$
\forall\left(f\left(x_{1}, \ldots, x_{n}\right)=y \equiv D\right)
$$

is conservative over $N_{c}^{=}+U_{y} D$ where $U_{y} D$ states the uniqueness condition for $y$ satisfying $D$, namely, $\forall\left(D \wedge D\left\{y / y^{\prime}\right\} \rightarrow y^{\prime}=y\right)$.

Proof Under $U_{y} D, D \wedge \forall y^{\prime}\left(D\left\{y / y^{\prime}\right\} \equiv y^{\prime}=y\right)$ and $D$ are obviously logically equivalent, so that it suffices to substitute the latter for the former in $\iota_{y}(f: D)$, in Theorem 1.2.

Directions for further work As we noted, the notion of strictness we have adopted is tailored to fit the proposal in [11], to deal with singular terms; hence it doesn't assume that if $t=t$ holds, then $t$ is denoting. It would be interesting to match the present treatment with the more demanding notion of strictness, by finding an appropriate axiomatization of equality. The $t v$-semantic approach to the conservativity of partial description functions and of partial selection functions, in the latter case under the assumption of the determinacy of equality, namely, the assumption $\forall x \forall y(x=y \vee x \neq y)$, should be extended to the case of intuitionistic logic. Obviously such questions call also for a proof-theoretic treatment. That requires a preliminary investigation of logic with equality and the proof of an appropriate subterm and subformula property (for cut free derivations in a suitable sequent calculus). Joint work with F. Previale in that direction is well under way.

\section{Notes}

1. So christened in [7, p. 128] and expressed by Quine's dictum from [14, p. 32], "to be is to be the value of a variable."

2. Leblanc found truth-value semantics to be a useful teaching device enabling students to grasp fundamental semantic concepts more easily, because it abstracted from tedious details in standard, set-theoretic semantics.

3. The concept of a formula is ordinarily used in a more general sense; the special case defined (above) might thus perhaps be described as a purely logical formula.

4. Note that none of the rules and relations concerning $\triangleright_{\mathrm{pc}}$ which are being used are specific to classical logic.

\section{References}

[1] Belnap, N. D., Jr., and G. J. Massey, "Semantic holism," Studia Logica, vol. 49 (1990), pp. 67-82. MR 1078439. DOI 10.1007/BF00401554. 385

[2] Feferman, S., "Definedness," pp. 295-320 in Varia, with a Workshop on the Foundations of Partial Functions and Programming (Irvine, Calif., 1995), vol. 43 of Erkenntnis, Kluwer, Dordrecht, 1995. MR 1396840. DOI 10.1007/BF01135376. 384, 391

[3] Gentzen, G., "Untersuchungen über das logische Schließen," Mathematische Zeitschrift, vol. 39 (1935), pp. 176-210, 405-31. 383, 385

[4] Gentzen, G., "Die Wiederspruchsfreiheit der reinen Zahlentheorie," Mathematische Annalen, vol. 112 (1936), pp. 493-565. MR 1513060. DOI 10.1007/BF01565428. 384 
[5] Gentzen, G., The Collected Papers of Gerhard Gentzen, edited by M. E. Szabo, Studies in Logic and the Foundations of Mathematics, North Holland, Amsterdam, 1969. Zbl 0209.30001. MR 0262050. 385

[6] Gumb, R., "Obituary of Hugues Leblanc," Bulletin of Symbolic Logic, vol. 6 (2000), pp. 230-31. 384

[7] Hintikka, J., "Existential presuppositions and existential commitments," Journal of Philosophy, vol. 56 (1959), pp. 125-37. 383, 394

[8] Leblanc, H., "Truth-value semantics for a logic of existence," Notre Dame Journal of Formal Logic, vol. 12 (1971), pp. 153-68. MR 0292640. 383

[9] Leblanc, H., Truth-Value Semantics, North-Holland, Amsterdam, 1976. MR 0457144. 383

[10] Leblanc, H., "Alternatives to standard first order semantics," pp. 53-131 in Handbook of Philosophical Logic, Vol. 2, 2nd ed., Kluwer, Dordrecht, 2001. MR 1884629. 383

[11] Leblanc, H., and T. Hailperin, "Nondesignating singular terms," Philosophical Review, vol. 68 (1959), pp. 239-43. 383, 387, 394

[12] Massey, G. J., “The pedagogy of logic: Humanistic dimensions,” Teaching Philosophy, vol. 4 (1981), pp. 303-36. 385

[13] Prawitz, D., Natural Deduction: A Proof-Theoretical Study, vol. 3 of Stockholm Studies in Philosophy, Almqvist and Wiksell, Stockholm, 1965. Zbl 0173.00205. MR 0193005. 383, 384

[14] Quine, W. V. O., "On what there is," Review of Metaphysics, vol. 2 (1948), pp. 21-38. 394

\section{Acknowledgments}

The author is grateful to Alberto Marcone and Flavio Previale for helpful conversations and remarks, and to the referees for very useful comments and suggestions.

Department of Mathematics and Computer Science

University of Udine

via Delle Scienze 206, 33100 Udine

Italy

franco.parlamento@uniud.it 